\title{
Internal and External Op-Amp Compensation: A Control-Centric Tutorial
}

\author{
Kent H. Lundberg \\ Department of Electrical Engineering and Computer Science \\ Massachusetts Institute of Technology, Cambridge, MA 02139 \\ email: klund@mit.edu
}

\begin{abstract}
Frequency compensation of two-stage integratedcircuit operational amplifiers is normally accomplished with a capacitor around the second stage. This compensation capacitance creates the desired dominant-pole behavior in the openloop transfer function of the op amp. Circuit analysis of this compensation leads to a mathematical observation of "pole splitting:" that as the compensation capacitance is increased, the parasitic poles of the amplifier separate in frequency.

Treatment of op-amp compensation as minor-loop feedback, instead of pole splitting, greatly simplifies and generalizes the analysis and design of op-amp frequency response. Using classical-control techniques instead of direct circuit analysis, insight and intuition into the behavior and flexibility of the system are gained.
\end{abstract}

\section{INTRODUCTION}

Operational amplifiers have been used by control engineers for many decades as key components in compensators [1], sensor circuitry [2], and analog computers [3], [4]. They are still one of the most ubiquitous electronic elements in the world. However, despite the required use of feedback in all opamp applications, and the presence of feedback in the internal circuitry, the design of operational amplifiers is often presented and completed without a useful control framework.

Op amps require a deliberately designed frequency response to ensure stability and satisfactory transient performance in end-user applications. Standard frequency compensation is designed for general-purpose op-amp applications such as amplifiers, buffers, and integrators. Sophisticated compensation techniques can be employed in specific applications in which standard compensation methods perform poorly.

Internally compensated op amps have a fixed transfer function set by the manufacturer. In the design of the circuit, the op-amp designer must choose a compensation network that is appropriate for the intended applications of the op amp. Externally compensated op amps [5] allow the end user to select the compensation network that determines the transfer function of the op amp. The determination and implementation of appropriate op-amp transfer functions in various applications is easily understood with the tools of classical control.

Popular textbooks in analog circuit design [6], [7], [8] treat op-amp compensation in a network-theory context, writing out many node equations and discussing the concept of "pole splitting" [9]. This approach is unnecessarily abstruse. Treatment

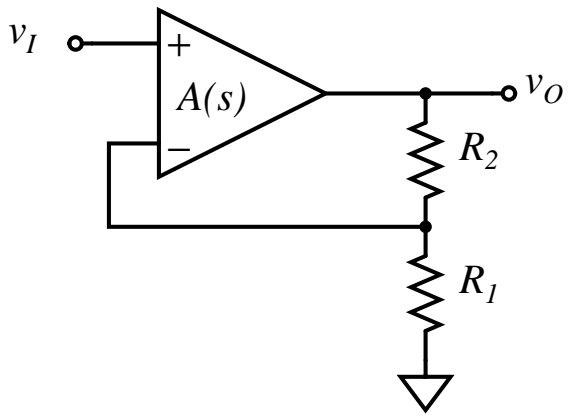

Fig. 1. Schematic for a simple non-inverting amplifier circuit. For generalpurpose use (and commercial success) this circuit must be stable for any resistor values $R_{1}$ and $R_{2}$.

of op-amp compensation as minor-loop feedback, instead of pole splitting, greatly simplifies and generalizes the analysis and design of op-amp frequency-compensation networks.

This paper demonstrates the use of classical-control techniques instead of direct circuit analysis in the design of compensation for general-purpose and special-purpose operational amplifiers. Intuition and insight into the solution are gained by using these feedback techniques.

\section{The General-Purpose Transfer Function}

The frequency response of general-purpose op amps is designed to be stable in the largest number of applications. The schematic for a simple non-inverting amplifier circuit is shown in Figure 1. This amplifier circuit is implemented with a negative-feedback loop around the op amp, and the closedloop gain is

$$
\frac{V_{o}}{V_{i}}=\frac{R_{1}+R_{2}}{R_{1}} .
$$

For general-purpose use the op amp must be designed such that this circuit is stable for any resistor values $R_{1}$ and $R_{2}$. The block diagram of this circuit is shown in Figure 2. The circuit loop transfer function is

$$
L(s)=A(s) \frac{R_{1}}{R_{1}+R_{2}}=A(s) F .
$$

For stability in this application, this loop transfer function must create a stable feedback system for any value of $F$ less than 


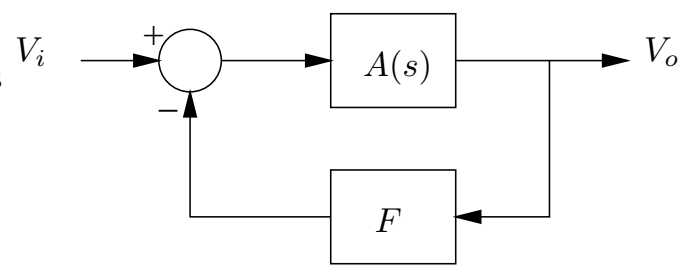

Fig. 2. Block diagram for the non-inverting amplifier circuit in Figure 1. The feedback path is $F=R_{1} /\left(R_{1}+R_{2}\right)$. The op-amp transfer function $A(s)$ must be designed to guarantee stability for any such attenuative feedback.

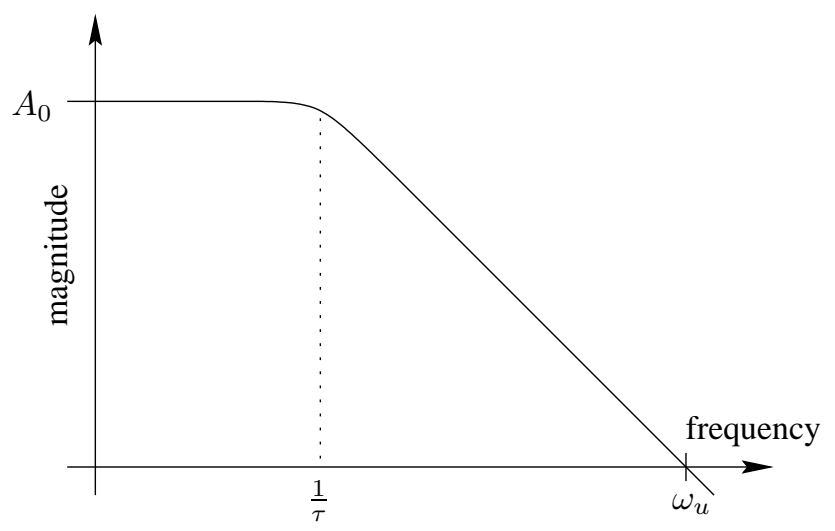

Fig. 3. Frequency response of the desired op-amp transfer function $A(s)$. The single-pole roll-off (slope of -1 ) behavior over a wide frequency range gives the desired transfer function for a general-purpose op amp. The frequency $\omega_{u}$ is the unity-gain frequency of the op amp.

one. The ideal transfer function that meets this requirement is

$$
A(s)=\frac{A_{0}}{\tau s+1} .
$$

With this op-amp transfer function, the closed-loop circuit will be stable for any choice of resistive feedback. The frequency response of this desired op-amp transfer function $A(s)$ rolls off with a slope of -1 over a wide frequency range, as shown in Figure 3. In the ideal case, this transfer function gives $90^{\circ}$ of phase margin, regardless of the feedback $F$.

A real op amp will have additional high-frequency poles beyond its unity-gain frequency $\omega_{u}$. Including the effect of an additional pole at $2 \omega_{u}$, the frequency response of the loop transfer function of the op-amp circuit with a variety of feedback terms is shown in Figure 4. Even with this additional high-frequency pole, the loop transfer function always crosses over with $60^{\circ}$ (or more) of phase margin for any attenuative feedback. Thus, stability is guaranteed for any set of feedback resistors.

The implementation of this desired op-amp transfer function is easier said than done. Even a simple op-amp circuit model gives an unacceptable op-amp transfer function.

For example, a simplified schematic of the Fairchild $\mu \mathrm{A} 741$ [10] op amp is shown in Figure 5. This circuit can be modeled by the equivalent-circuit block diagram shown in Figure 6 . The frequency response of this circuit, when uncompensated, is shown in Figure 7. The two low-frequency poles severely

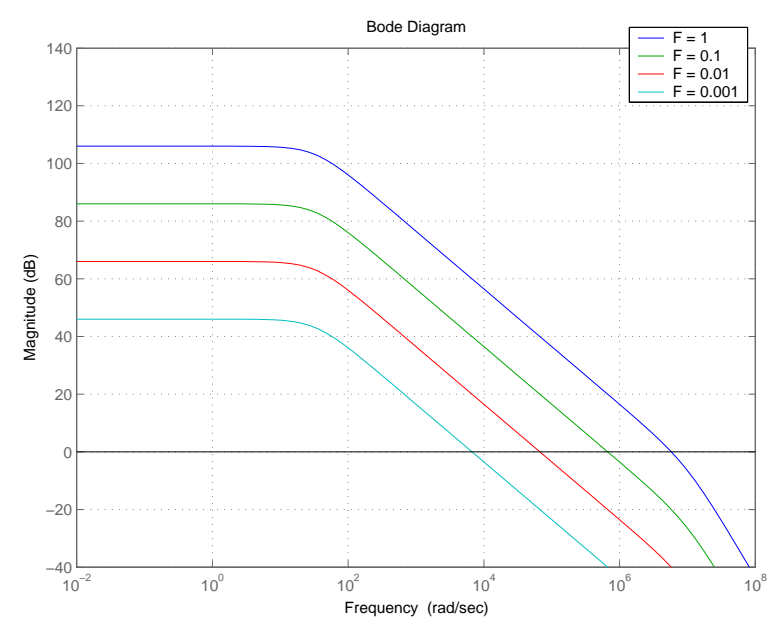

Fig. 4. Frequency response of the op-amp-circuit loop transfer function $L(s)$ with a variety of feedback terms. Since the loop transfer function always crosses over with $60^{\circ}$ or more of phase margin for any attenuative feedback, stability is guaranteed.

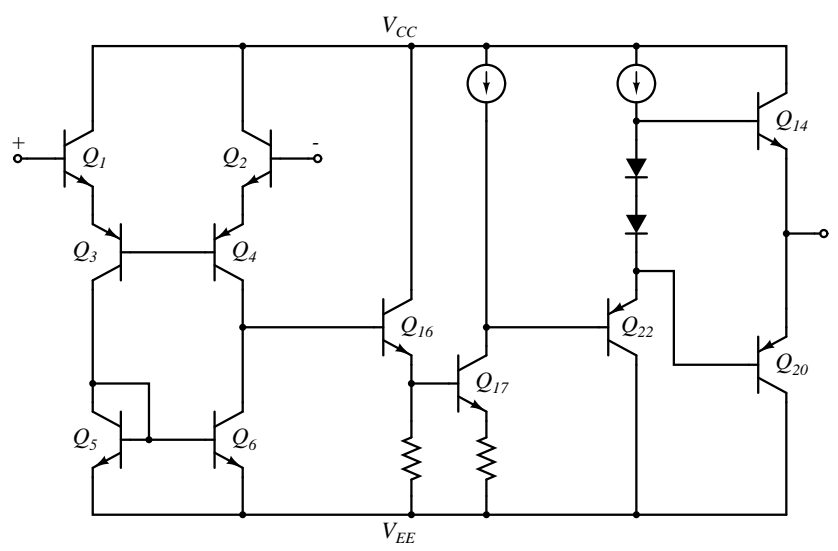

Fig. 5. Simplified schematic of the uncompensated Fairchild $\mu$ A741 op amp, showing the signal-path transistors. The full schematic is shown and explained in Appendix II.

degrade the phase margin at crossover. Additional highfrequency poles in the circuit make matters worse.

For stability in amplifier applications, the op amp must be compensated to achieve a frequency response similar to the ideal transfer function in equation (1) and shown in Figure 3. This general-purpose compensation is usually accomplished with a capacitor [5]. (This technique is often called "Miller compensation." See Appendix I.) The simplified schematic of the $\mu \mathrm{A} 741$ op amp with a compensation capacitor is shown in Figure 8. The compensation capacitor goes around the highgain stage as shown in the equivalent-circuit block diagram in Figure 9.

Using two-port circuit models for each stage, the equivalentcircuit schematic in Figure 10 can be drawn. Each gain stage is represented by a Norton-equivalent two-port model with input resistance, output resistance, output capacitance, and a transconductance generator. The output buffer is ignored in this equivalent circuit since the output voltage of the 


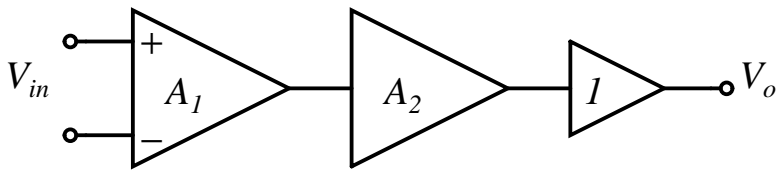

Fig. 6. Equivalent-circuit block diagram of a two-stage op amp. The input stage $A_{1}$ converts the input signal from differential to single-ended. The second stage $A_{2}$ is the high-gain stage. The output buffer provides current gain and protection at the output.

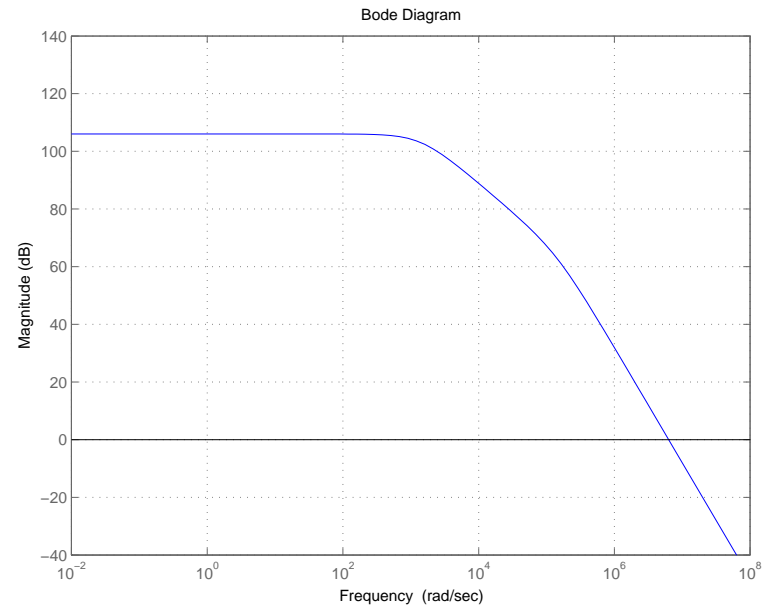

Fig. 7. Frequency response of an uncompensated op amp. The two lowfrequency poles in the uncompensated transfer function severely degrade the phase margin at crossover.

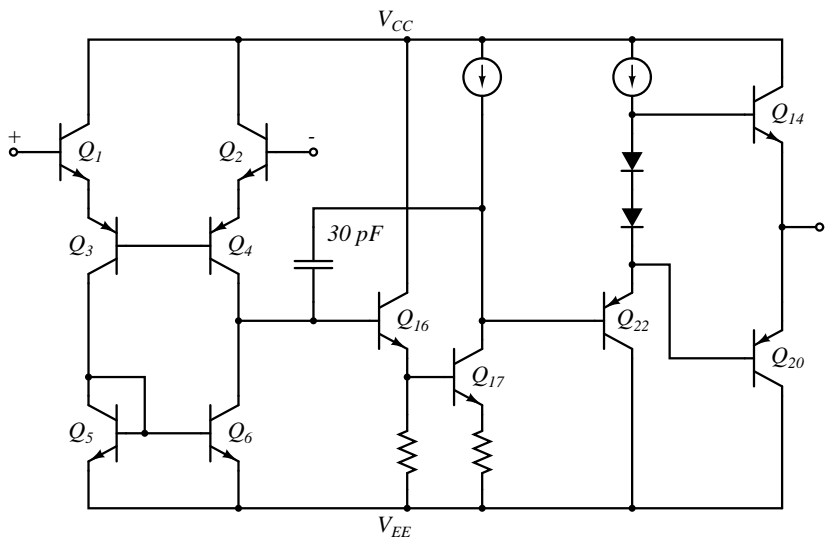

Fig. 8. Simplified schematic of the Fairchild $\mu$ A741 op amp with compensation capacitor. The compensation capacitor goes around the high-gain second stage created by $Q_{16}$ and $Q_{17}$.

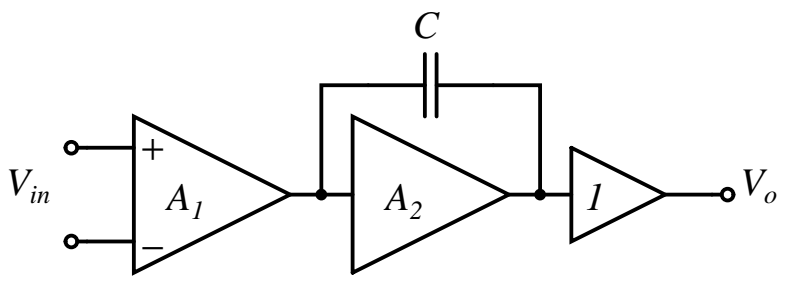

Fig. 9. Equivalent-circuit block diagram of a two-stage op amp with compensation capacitor. The compensation capacitor goes around the highgain second stage.



Fig. 10. Equivalent-circuit schematic for the two-stage op amp with compensation capacitor of Figure 9 , where $A_{1}=G_{M 1} R_{1}$ and $A_{2}=G_{M 2} R_{2}$.

second stage is equal to the buffer output voltage $V_{o}$. The transfer function of this equivalent circuit will be derived in the following sections, using the pole-splitting approach in Section III and using a feedback approach in Section IV.

\section{POle-SPlitTing APPROACH}

To investigate the effects of the compensation capacitor, the transfer function of the op-amp equivalent-circuit schematic in Figure 10 is calculated to find

$$
A(s)=\frac{V_{o}}{V_{\text {in }}}(s) .
$$

The pole-splitting approach [9] uses brute-force circuit analysis to determine this transfer function. The approach starts with the constitutive current equations at the two circuit nodes $V_{1}$ and $V_{o}$

$$
\begin{aligned}
& G_{M 1} V_{i n}-\frac{V_{1}}{R_{1}}-s C_{1} V_{1}-s C\left(V_{1}-V_{o}\right)=0 \\
& s C\left(V_{1}-V_{o}\right)-G_{M 2} V_{1}-\frac{V_{o}}{R_{2}}-s C_{2} V_{o}=0 .
\end{aligned}
$$

After a page of algebra (as shown in detail in Appendix III) the transfer function is found

$$
A(s)=\frac{V_{o}}{V_{\text {in }}}(s)=\frac{G_{M 1} R_{1} G_{M 2} R_{2}\left(C s / G_{M 2}-1\right)}{a_{2} s^{2}+a_{1} s+1}
$$

where the coefficients of the denominator are

$$
\begin{aligned}
& a_{2}=R_{1} R_{2}\left(C_{1} C_{2}+C_{1} C+C_{2} C\right) \\
& a_{1}=R_{1} C_{1}+R_{1} C+R_{2} C_{2}+R_{2} C+G_{M 2} R_{2} R_{1} C .
\end{aligned}
$$

Assuming that the gain of the second stage is large $\left(G_{M 2} R_{2} \gg 1\right)$, the final term in the first-order coefficient $a_{1}$ dominates the sum, and the transfer function can be simplified as

$$
\begin{aligned}
& A(s) \approx \\
& \quad \frac{G_{M 1} R_{1} G_{M 2} R_{2}\left(C s / G_{M 2}-1\right)}{R_{1} R_{2}\left(C_{1} C_{2}+C C_{1}+C C_{2}\right) s^{2}+G_{M 2} R_{2} R_{1} C s+1} .
\end{aligned}
$$

The locations of the transfer-function poles can be found by assuming that the pole locations are widely separated

$$
A(s) \approx \frac{A_{0}}{\left(\tau_{1} s+1\right)\left(\tau_{2} s+1\right)}=\frac{A_{0}}{\tau_{1} \tau_{2} s^{2}+\left(\tau_{1}+\tau_{2}\right) s+1} .
$$

If the two poles are widely separated $\left(\tau_{1} \gg \tau_{2}\right)$, then

$$
A(s) \approx \frac{A_{0}}{\tau_{1} \tau_{2} s^{2}+\tau_{1} s+1} .
$$




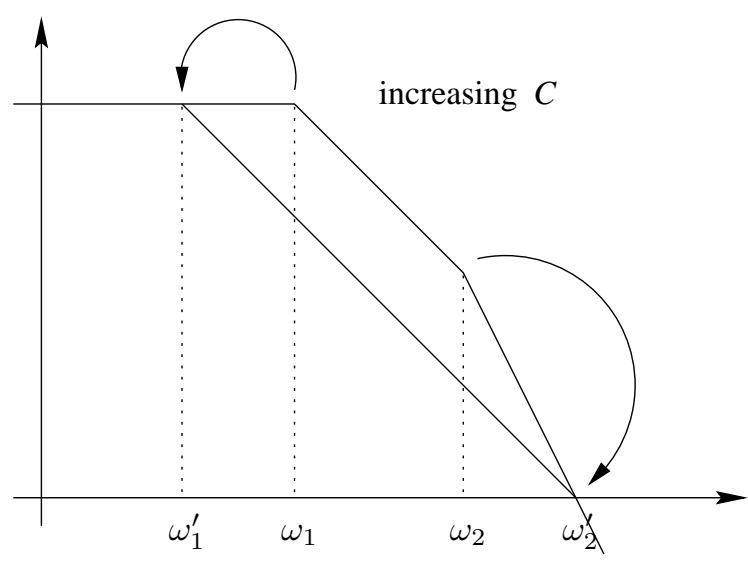

Fig. 11. The mathematical observation of "pole splitting." As the size of the compensation capacitor $C$ is increased, the frequency of the first pole $\omega_{1}$ decreases and the frequency of the second pole $\omega_{2}$ increases. The poles apparently "split" in frequency.

Therefore, the approximate pole locations of the op-amp transfer function are

$$
\begin{aligned}
& \omega_{1}=\frac{1}{\tau_{1}}=\frac{1}{a_{1}}=\frac{1}{G_{M 2} R_{2} R_{1} C} \\
& \omega_{2}=\frac{\tau_{1}}{\tau_{1} \tau_{2}}=\frac{a_{1}}{a_{2}}=\frac{G_{M 2} C}{C_{1} C_{2}+C C_{1}+C C_{2}} .
\end{aligned}
$$

Figure 11 shows the resulting "pole-splitting" behavior in the frequency response of this transfer function. It is observed that as the size of the compensation capacitor is increased, the low-frequency pole location $\omega_{1}$ decreases in frequency, and the high-frequency pole $\omega_{2}$ increases in frequency. The poles appear to "split" in frequency. For a large enough compensation capacitor, a single-pole roll off over a wide range of frequency results, as shown in Figure 11, which matches the desired transfer function in Figure 3.

\section{Minor-LoOP FEEDBACK}

While the above results are correct and useful, they are an impediment to intuition [11]. Treating the compensation capacitor $C$ as a minor-loop feedback path, instead of as just another circuit element, simplifies the analysis of the compensated op amp. The concept of op-amp compensation by minor-loop feedback provides useful design insight into the flexibility of this topology and opens up a wide range of applications for special-purpose compensation schemes.

In the minor-loop approach, the capacitor $C$ is treated as a feedback path as shown in Figure 12. If the gain of the second stage is large, then the first-stage voltage $V_{1}$ will be much smaller than the second-stage voltage $V_{o}$. Comparatively, the node $V_{1}$ appears to be a virtual ground. Therefore the effect of the capacitor can be modeled as an admittance $Y_{c}(s)$ that injects a current $I_{c}$ into the first stage that depends only on the voltage of the second stage $V_{o}$

$$
I_{c}=Y_{c}(s) V_{o}=s C V_{o}
$$

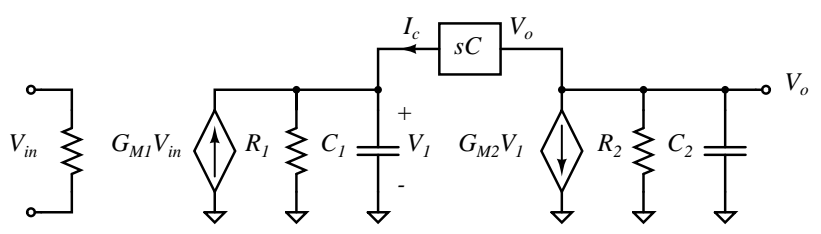

Fig. 12. Equivalent-circuit schematic for the op amp with the compensation capacitor modeled as a block. The admittance of the capacitor injects a current $I_{c}=s C V_{o}$ into the output node of the first stage.

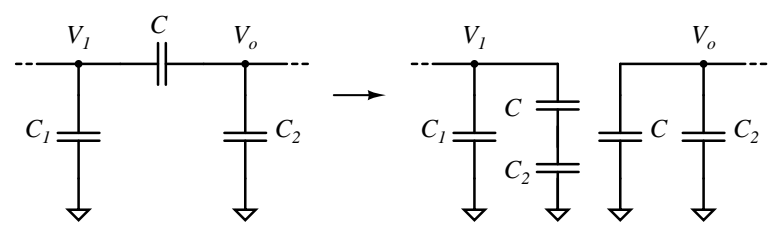

Fig. 13. High-frequency model of the effective capacitive loading by the compensation capacitor. When the compensation capacitor $C$ is removed from the circuit at left, the circuit is transformed into the circuit at right so that the capacitive loading on each stage is maintained.

When the compensation capacitor $C$ is removed from the circuit and replaced with this block, the capacitive loading on each stage must be maintained. A high-frequency model of the effective capacitive loading of the compensation capacitor is shown in Figure 13. Therefore the capacitors $C_{1}$ and $C_{2}$ in Figure 12 are replaced with the capacitors $C_{3}$ and $C_{4}$, where

$$
C_{3}=C_{1}+\frac{C_{2} C}{C_{2}+C}
$$

and

$$
C_{4}=C_{2}+C \text {. }
$$

In the equivalent circuit in Figure 12, the voltage $V_{1}$ is the total current flowing into the first node times the impedance of $R_{1}$ and $C_{3}$

$$
V_{1}=\left(G_{M 1} V_{i n}+I_{c}\right)\left(\frac{R_{1}}{R_{1} C_{3} s+1}\right) .
$$

The voltage $V_{o}$ is the current flowing into the second stage times the impedance of $R_{2}$ and $C_{4}$

$$
V_{o}=-G_{M 2} V_{1}\left(\frac{R_{2}}{R_{2} C_{4} s+1}\right) .
$$

From equations (6) and (7) the block diagram of the equivalent circuit with minor-loop feedback can be drawn, as shown in Figure 14. The block diagram can be rearranged into Figure 15 by pushing the $G_{M 1}$ block inside the loop.

As shown in Figure 15, the forward path of the op amp is

$$
G(s)=\left(\frac{G_{M 1} R_{1}}{R_{1} C_{3} s+1}\right)\left(\frac{G_{M 2} R_{2}}{R_{2} C_{4} s+1}\right)
$$

and the feedback path is

$$
H(s)=\frac{Y_{c}(s)}{G_{M 1}}=\frac{s C}{G_{M 1}} .
$$




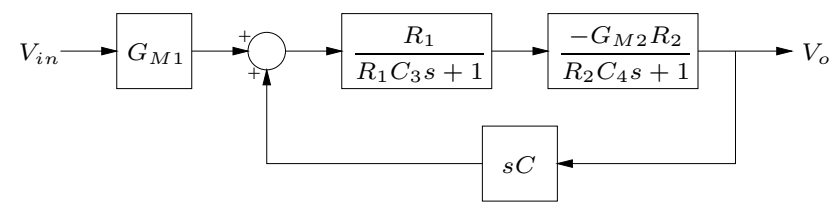

Fig. 14. Block diagram of the equivalent circuit with the compensation capacitor shown as a minor-loop feedback block. This block diagram is drawn from the node equations (6) and (7).

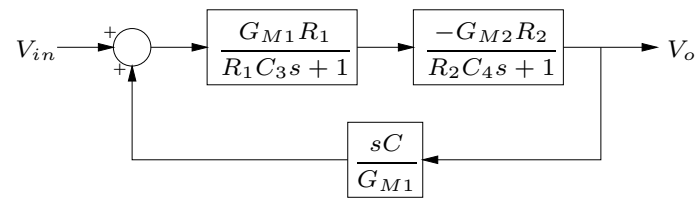

Fig. 15. Equivalent block diagram of the op amp, with the outside block from Figure 14 moved inside the minor loop. This block diagram produces the forward-path and feedback-path transfer functions (8) and (9).

The transfer function of the op amp can now be calculated from this block diagram with Black's formula

$$
A(s)=\frac{V_{o}}{V_{\text {in }}}(s)=\frac{G(s)}{1+G(s) H(s)}
$$

or it can be determined from $G(s)$ and $1 / H(s)$ on the asymptotic Bode plot shown in Figure 16 (as explained in Appendix IV).

An accurate rendering of the Bode magnitude plot of the forward-path and the inverse-feedback-path transfer functions is shown in Figure 17. The Bode magnitude plot of the resulting op-amp transfer function $A(s)$ is shown in Figure 18.

Finally, the locations of the resulting poles of the op-amp transfer function $A(s)$ can be found from the intersections of the two curves in Figure 16. The low-frequency intersection occurs when the low-frequency behavior of the forward path (below the frequencies its poles) intersects with the inverse of the feedback path. At low frequency

$$
\lim _{\omega \rightarrow 0}|G(j \omega)|=G_{M 1} R_{1} G_{M 2} R_{2}
$$

therefore, the intersection occurs when

$$
G_{M 1} R_{1} G_{M 2} R_{2}=\frac{G_{M 1}}{\omega C}
$$

at a frequency of

$$
\omega_{1}=\frac{1}{R_{1} G_{M 2} R_{2} C} .
$$

This result agrees exactly with the result (4) found in Section III using brute-force circuit analysis.

The high-frequency intersection occurs when the highfrequency behavior of the forward path intersects with the inverse of the feedback path. At high frequency

$$
\lim _{\omega \rightarrow \infty}|G(j \omega)|=\frac{G_{M 1} G_{M 2}}{\omega^{2} C_{3} C_{4}}
$$

therefore, the intersection occurs when

$$
\frac{G_{M 1} G_{M 2}}{\omega^{2} C_{3} C_{4}}=\frac{G_{M 1}}{\omega C}
$$

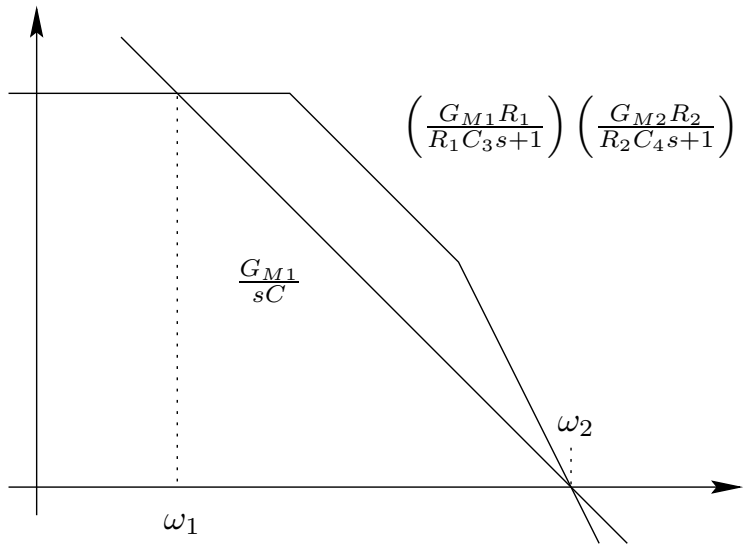

Fig. 16. Asymptotic Bode plot showing $G(s)$ and $1 / H(s)$ for the op amp from the block diagram in Figure 15. The asymptotic op-amp transfer function $A(s)$ is simply the lower of these two curves.

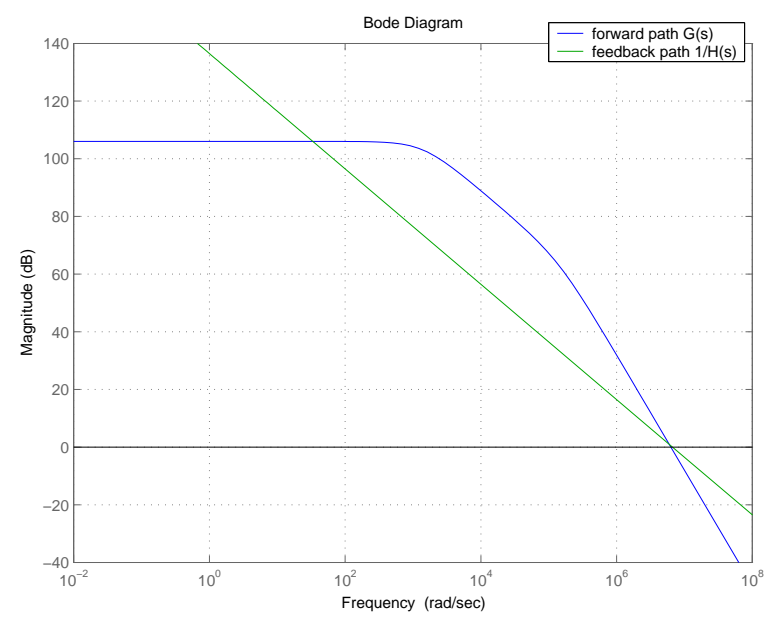

Fig. 17. Bode magnitude plot of the forward-path $G(s)$ and inverse-feedbackpath $1 / H(s)$ transfer functions from the block diagram in Figure 15. This plot is a more accurate rendering of the asymptotic plot in Figure 16.

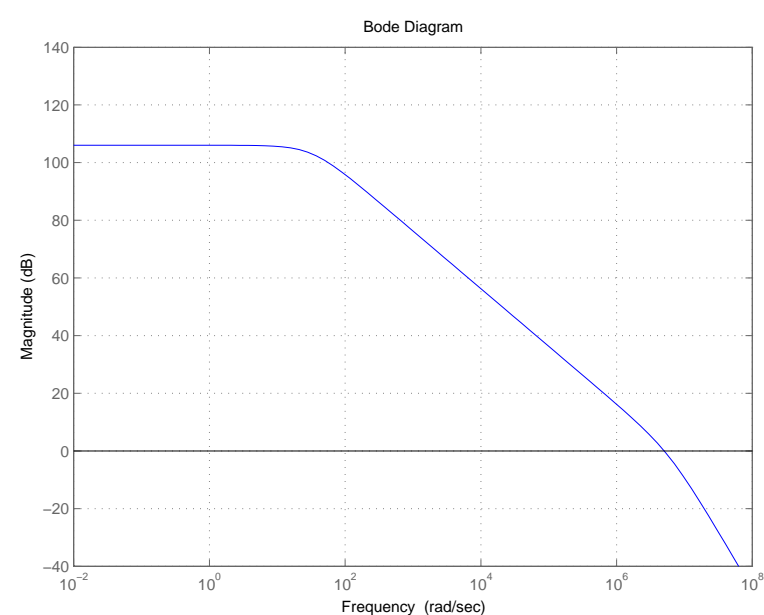

Fig. 18. Bode magnitude plot of the closed-minor-loop transfer function of the op amp $A(s)$. This plot is the lower of the two curves from Figure 17 and matches the desired transfer function in Figures 3 and 4. 


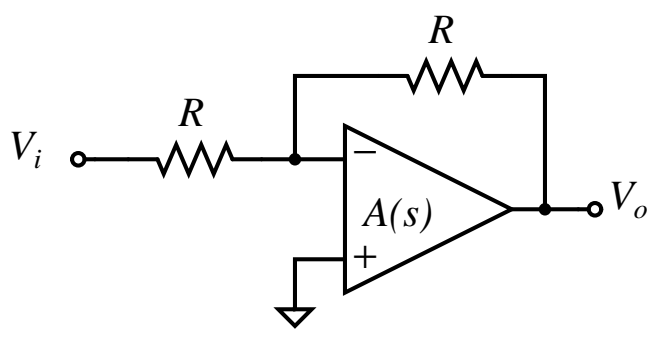

Fig. 19. Schematic for an inverting gain-of-one op-amp amplifier.

at a frequency of

$$
\omega_{2}=\frac{G_{M 2} C}{C_{3} C_{4}}
$$

where

$$
C_{3} C_{4}=C_{1} C_{2}+C_{1} C+C_{2} C .
$$

Again, this result agrees exactly with the result (5) found above using brute-force circuit analysis.

Using this minor-loop feedback approach to calculating the compensated transfer function of the op amp produced the same results with less work. In addition, a better understanding of the internals of the op amp is achieved. The minor-loop feedback path created by the compensation capacitor (or the compensation network) allows the frequency response of the op-amp transfer function to be easily shaped.

\section{COMPEnsation for Steady-STATE ERror}

This feedback approach to op-amp compensation can be exploited in the design of special-purpose op-amp transfer functions. Such transfer functions can be used to improve the performance characteristics of many op-amp circuits. Using these minor-loop techniques, these special-purpose op-amp transfer functions are easier to design.

For example, the dynamic-tracking behavior of an op-amp amplifier circuit can be modified with appropriate changes to the op-amp transfer function $A(s)$. An inverting op-amp amplifier is shown in Figure 19. With standard capacitive compensation, the steady-state error to a step input is nearly zero, since the op-amp transfer function looks like an integrator

$$
A(s) \approx \frac{G_{M 1}}{Y_{c}(s)}=\frac{G_{M 1}}{s C}
$$

with a single pole near the origin. If zero steady-state error to a ramp input is desired, the transfer function of the op amp can be changed to achieve this specification. As seen in Section IV, the op-amp transfer function can be designed by appropriate choice of the compensation network. An op-amp transfer function such as

$$
A(s) \approx \frac{G_{M 1}(\tau s+1)}{C \tau s^{2}}
$$

can be achieved with a compensation admittance of the form

$$
Y_{c}(s)=\frac{C \tau s^{2}}{\tau s+1} .
$$

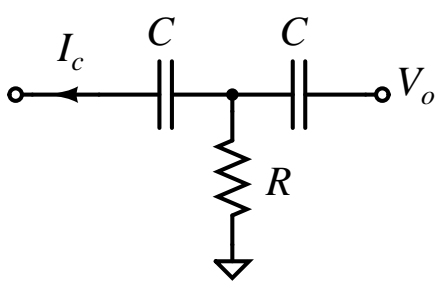

Fig. 20. Two-port compensation network to replace the compensation capacitor in Figure 10. The capacitor terminals connect around the highgain stage, and the resistor connects to ground. The transfer admittance $Y_{c}(s)=I_{c} / V_{o}$ of this network has two zeros at the origin.

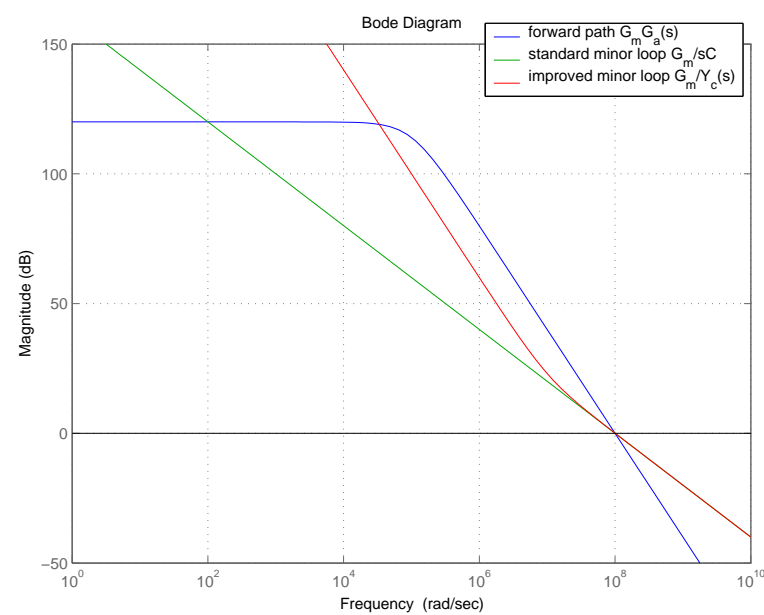

Fig. 21. Bode magnitude plot of the forward-path and inverse-feedback-path transfer functions for single-pole and two-pole compensation.

This double-integrator transfer function will exhibit zero steady-state error to an input ramp.

The transfer admittance of the two-port compensation network shown in Figure 20 has two zeros at the origin

$$
Y_{c}(s)=\frac{I_{c}}{V_{o}}=\frac{R C^{2} s^{2}}{2 R C s+1} .
$$

Therefore, with this compensation, the open-loop transfer function of the op amp is approximately

$$
A(s) \approx \frac{G_{M 1}(2 R C s+1)}{R C^{2} s^{2}}
$$

with two poles at the origin. This "two-pole" compensation network [12] creates a slope of -2 in the frequency response of the op-amp transfer function.

A Bode magnitude plot of the forward-path and inversefeedback-path transfer functions for standard single-pole compensation and two-pole compensation is shown in Figure 21. A comparison of the resultant op-amp transfer functions is shown in Figure 22. The open-loop gain of the two-pole opamp transfer function exceeds the gain for the single-pole transfer function for all frequencies between $10^{2}$ and $10^{7}$ radians per second (rps). The response of the error signal for a fast input ramp of $1 \mathrm{~V} / \mu \mathrm{s}$ is shown in Figure 23. The increased gain of the two-pole op-amp transfer function significantly 


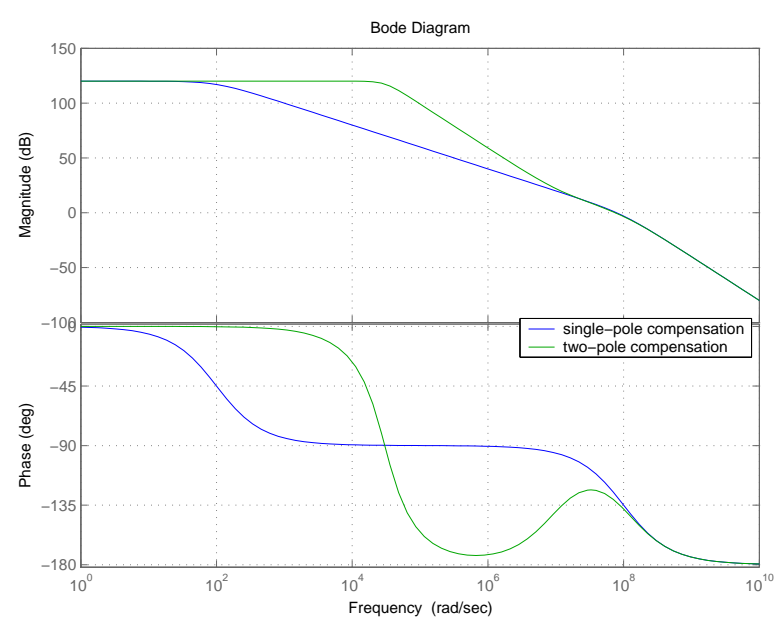

Fig. 22. Comparison of the single-pole and two-pole op-amp transfer functions $A(s)$. The open-loop gain of the two-pole op-amp transfer function exceeds the gain for the single-pole transfer function for all frequencies between $10^{2}$ and $10^{7} \mathrm{rps}$.



Fig. 23. Response of the error signal for a fast input ramp. The increased gain of the two-pole op-amp transfer function significantly reduces the magnitude of the steady-state error. The error signal for the two-pole compensated system is always smaller than for the single-pole op amp.

reduces the magnitude of the steady-state error, and this opamp transfer function is easier to design using minor-loop feedback techniques.

\section{COMPENSATION FOR CAPACITIVE LOADS}

This feedback approach to op-amp design can also illuminate and diagnose subtle problems in compensation network design. Some special-purpose op-amp transfer-function designs exhibit insidious stability problems that can be difficult to diagnose using only circuit analysis techniques.

\section{A. Compensation that Introduces a Zero}

Consider an op-amp unity-gain buffer circuit with capacitive load, as shown in Figure 24. The output resistance of the op



Fig. 24. Schematic for a unity-gain buffer circuit with a capacitive load The output resistance and the capacitive load create a low-pass filter in the feedback loop. There is no way to feedback from output to input without including this pole.

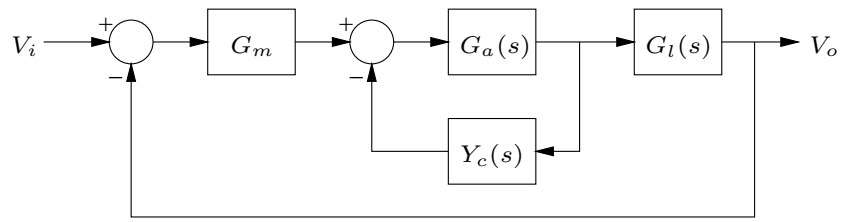

Fig. 25. Block diagram for the buffer in Figure 24 showing the minor-loop topology of the two-stage op amp. The low-frequency pole from the capacitive load appears between the second stage of the op amp and the output node of the circuit.

amp and the capacitance of the load create a low-pass filter in the feedback loop

$$
L(s)=\frac{A(s)}{R_{O} C_{L} s+1} .
$$

Because the output resistance is inside the op amp, feedback from the output terminal always includes the effects of this additional pole. The block diagram of the buffer circuit showing the minor loop of the op amp is shown in Figure 25. The low-frequency pole from the capacitive load appears between the second stage of the op amp and the output node of the circuit.

As an example, consider the op-amp model in Figure 12 with an input-stage transconductance of

$$
G_{m}=G_{M 1}=10^{-4} \mho,
$$

a second-stage transresistance of

$$
G_{a}(s)=\frac{R_{1} G_{M 2} R_{2}}{\left(R_{1} C_{1} s+1\right)\left(R_{2} C_{2} s+1\right)}=\frac{10^{10} \Omega}{\left(10^{-5} s+1\right)^{2}},
$$

and standard capacitive compensation of

$$
Y_{c}(s)=s C=10^{-11} s \mho .
$$

The Bode plot of the forward-path and inverse-feedback-path transfer functions for this op amp is shown in Figure 26. With this choice of $Y_{c}(s)$, the high-frequency pole of the op amp occurs two orders of magnitude above crossover.

The op-amp transfer function is

$$
A(s)=\frac{G_{m} G_{a}(s)}{1+G_{a}(s) Y_{c}(s)} \approx \frac{G_{m}}{Y_{c}(s)}=\frac{10^{7}}{s} .
$$

The Bode plot of the op-amp transfer function is shown in Figure 27. This transfer function $A(s)$ has nearly ninety 


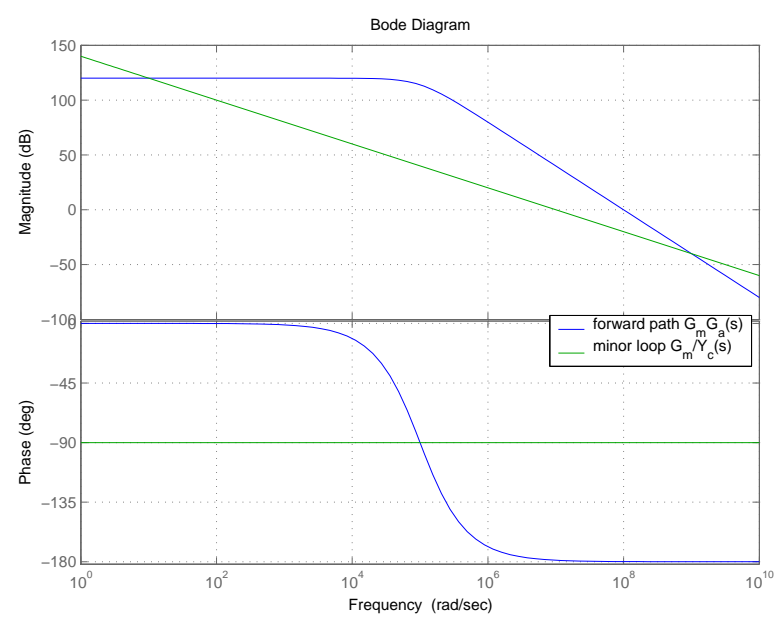

Fig. 26. Bode plot of the forward-path and inverse-feedback-path transfer functions for the example op amp with standard compensation. This op amp appears to be over-compensated since the second pole of the op amp occurs two orders of magnitude above crossover.



Fig. 27. Bode plot of the closed-minor-loop transfer function of the op amp $A(s)$. This transfer function has nearly ninety degrees of phase margin at unity-gain crossover. This op amp is over-compensated and is much more stable than it would need to be for most applications.

degrees of phase margin at crossover. This op amp is much more stable than it needs to be for most applications. The step response of the op-amp buffer (without capacitive load) is shown in Figure 28. With ninety degrees of phase margin, the response is first order.

However, with a finite output resistance and a capacitive load on the output $\left(R_{O}=10 \Omega\right.$ and $\left.C_{L}=0.1 \mu \mathrm{F}\right)$, the stability of the op amp is severely compromised. The transfer function due to the load is

$$
G_{l}(s)=\frac{1}{R_{O} C_{L} s+1}=\frac{1}{10^{-6} s+1} .
$$

With this capacitive load, the loop transfer function of the

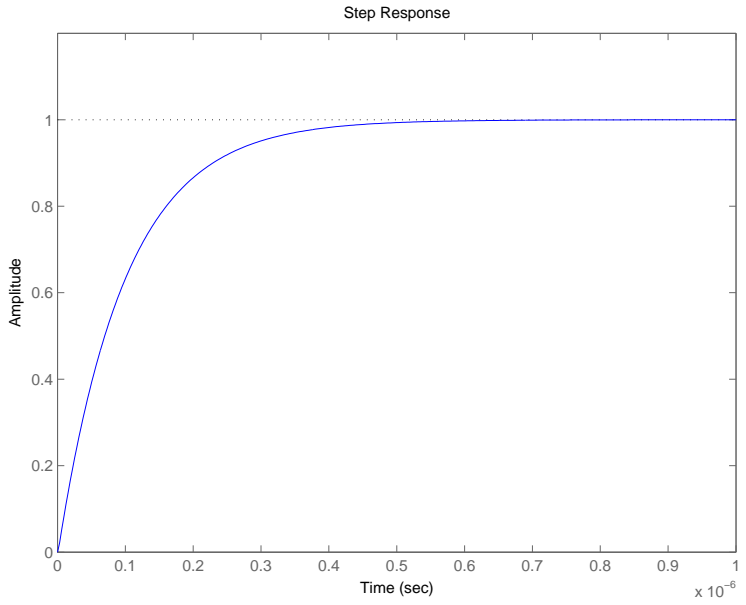

Fig. 28. Step response of the op-amp buffer without the capacitive load. With ninety degrees of phase margin, the response is first order.

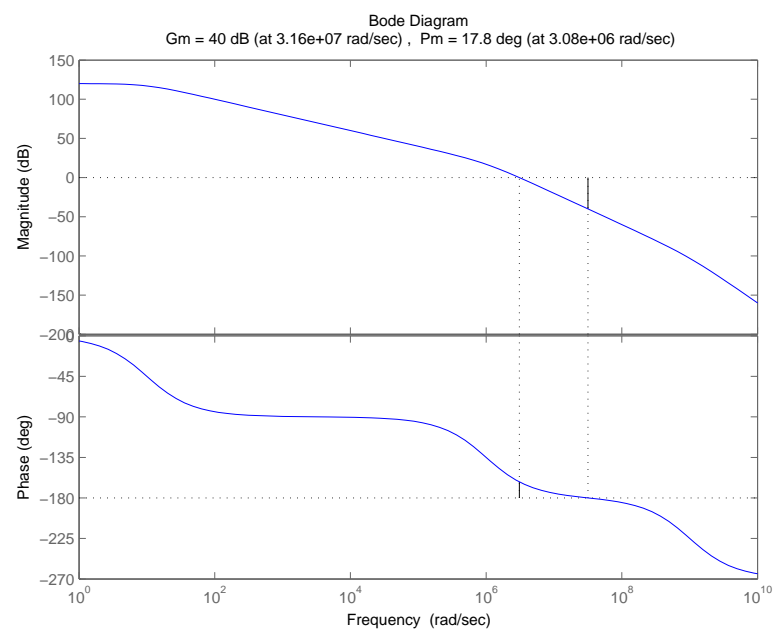

Fig. 29. Bode plot of the loop transfer function $L(s)$ for the op-amp buffer with capacitive load. The pole due to the capacitive load occurs below crossover, and stability is significantly compromised. The load capacitor reduces the phase margin from ninety degree (as shown in Figure 27) to less than eighteen degrees shown here.

whole circuit is approximately

$$
L(s)=A(s) G_{l}(s) \approx \frac{G_{m} G_{l}(s)}{Y_{c}(s)}=\frac{10^{7}}{s\left(10^{-6} s+1\right)} .
$$

The Bode plot of the loop transfer function for the op-amp circuit with capacitive load is shown in Figure 29. The pole due to the capacitive load occurs below crossover, so the loop transfer function crosses over with a slope of -2 and a small phase margin. Stability of the circuit is significantly compromised and the phase margin has decreased from ninety degrees to less than eighteen degrees.

This reduction in phase margin degrades the transient performance of the circuit. The step response of the op-amp buffer with capacitive load is shown in Figure 30. Clearly, the response is no longer first order. With less than eighteen degrees of phase margin, the response exhibits considerable 


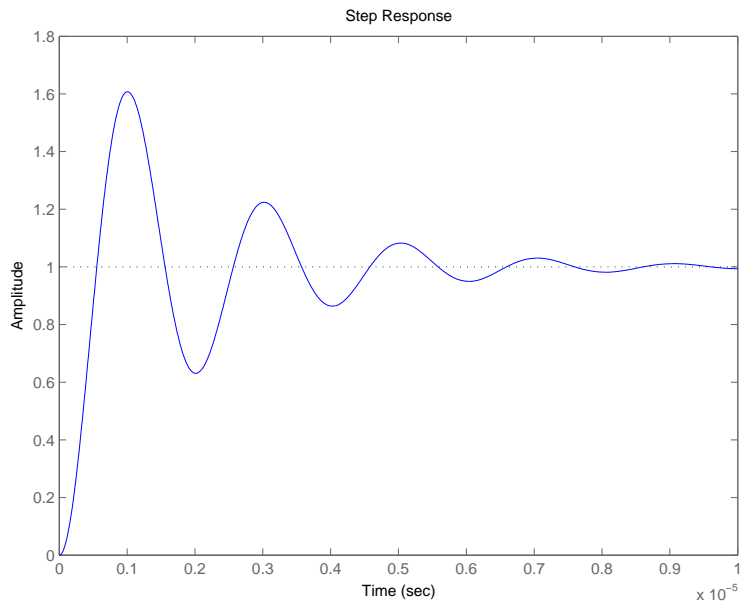

Fig. 30. Step response of the op-amp buffer with capacitive load. With less than eighteen degrees of phase margin, the response exhibits considerable peak overshoot and ringing.

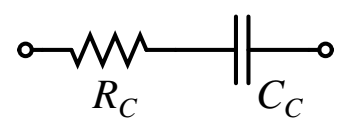

Fig. 31. Compensation network with an admittance pole. This pole in the feedback-path $Y_{c}(s)$ introduces a zero into the op-amp transfer function $A(s)$. This zero will be used to cancel the capacitive-load pole.

peak overshoot and ringing. In general, most op amps behave badly with a capacitive load.

Using the minor-loop feedback approach to op-amp compensation from Section IV to shape the transfer function, the op amp can be redesigned to compensate for the capacitive load. The op-amp transfer function $A(s)$ is changed to include a zero near the frequency of the additional pole in the circuit loop transfer function $L(s)$.

A compensation network that introduces a zero in the op-amp transfer function [13] is shown in Figure 31. The admittance of this network is

$$
Y_{c}(s)=\frac{I_{c}}{V_{o}}=\frac{C_{C} s}{R_{C} C_{C} s+1} .
$$

The pole in the admittance $Y_{c}(s)$ becomes a zero in the opamp transfer function $A(s)$. This zero will be used to cancel the effects of the capacitive load at the output. With this compensation network the op-amp transfer function is

$$
A(s) \approx \frac{G_{m}}{Y_{c}(s)}=\frac{G_{m}\left(R_{C} C_{C} s+1\right)}{C_{C} s}
$$

and the loop transfer function of the circuit is

$$
L(s)=A(s) G_{l}(s) \approx \frac{G_{m}\left(R_{C} C_{C} s+1\right)}{C_{C} s}\left(\frac{1}{R_{O} C_{L} s+1}\right) .
$$

If the time constant of the admittance is chosen to be equal to the time constant of the output pole $\left(R_{C} C_{C} \approx R_{O} C_{L}\right)$ then the term from the minor loop and the term from the capacitive load will cancel, and the loop transfer function returns to single-pole roll-off behavior.

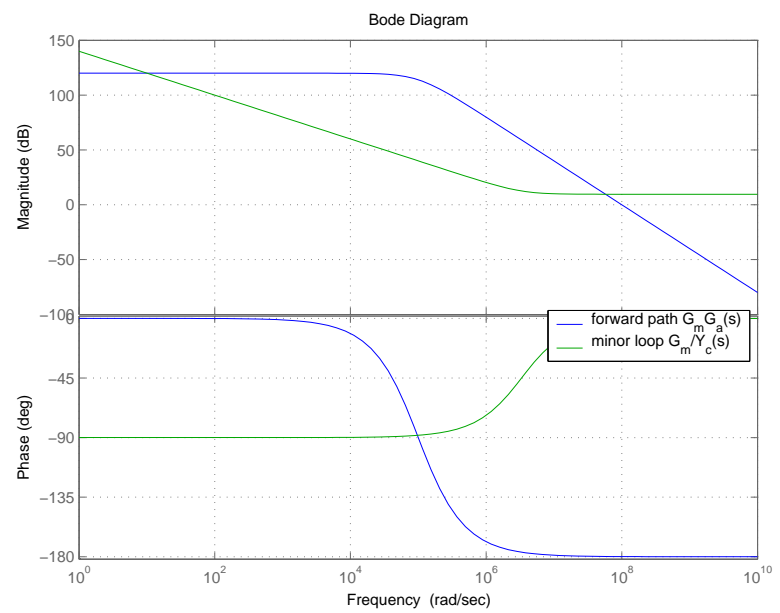

Fig. 32. Bode plot of the forward-path and inverse-feedback-path transfer functions for the op amp with compensation that introduces a zero. The pole in $Y_{c}(s)$ becomes a zero in $A(s)$.

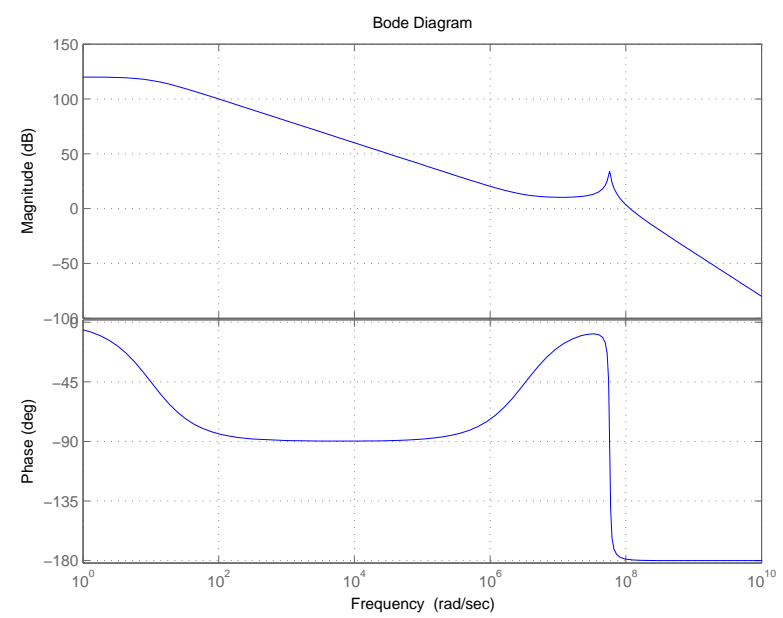

Fig. 33. Bode plot of the closed-minor-loop transfer function of the op amp $A(s)$. The transfer-function zero introduced by the compensation network is clearly visible near $3 \cdot 10^{6}$ rps. The resonance is blithely ignored.

For this example, the minor-loop transfer admittance is

$$
Y_{c}(s)=\frac{10^{-11} s \mho}{3 \cdot 10^{-7} s+1}
$$

and the circuit loop transfer function is approximately

$$
L(s) \approx \frac{10^{7}\left(3 \cdot 10^{-7} s+1\right)}{s}\left(\frac{1}{10^{-6} s+1}\right)
$$

(the $R C$ products are chosen to be slightly different to remain distinct on the Bode plots and to be a bit more realistic). The Bode plot of the forward-path and inverse-feedback-path transfer functions for the op amp with this compensation is shown in Figure 32. The Bode plot of the closed-minor-loop transfer function of the op amp $A(s)$ is shown in Figure 33. The transfer function zero is clearly visible at $3 \cdot 10^{6} \mathrm{rps}$.

The Bode plot of the loop transfer function for the op-amp circuit with capacitive load is shown in Figure 34. The zero 


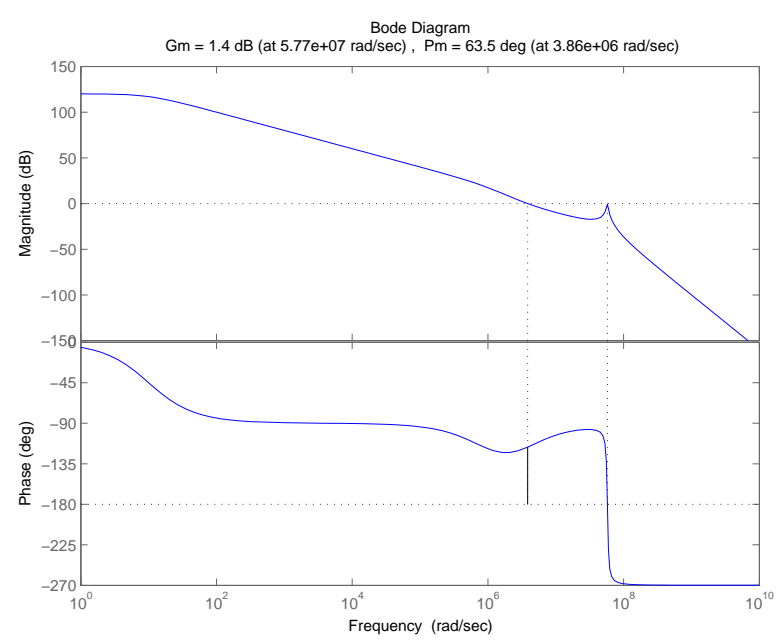

Fig. 34. Bode plot of the loop transfer function $L(s)$ for the op-amp buffer with capacitive load. The zero from the compensation network nearly cancels the pole from the capacitive load. The phase margin has increased from eighteen degrees in Figure 29 to more than sixty degrees.

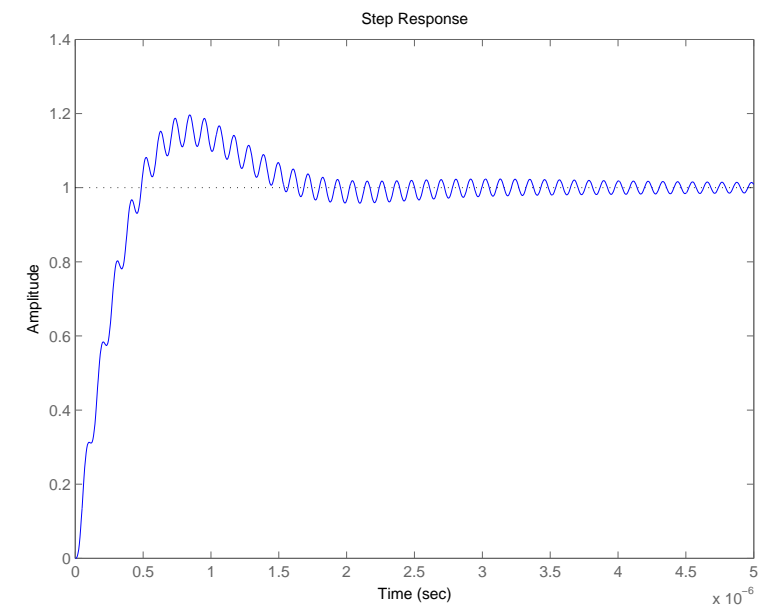

Fig. 35. Step response of the compensated op-amp buffer with capacitive load. The peak overshoot is greatly improved from Figure 30, but the highfrequency ringing indicates that the system is on the edge of instability.

from the compensation network nearly cancels the pole due to the capacitive load. The phase margin has increased from eighteen degrees to more than sixty degrees.

However, the step response shows a problem. The step response of the compensated op-amp buffer with capacitive load is shown in Figure 35. While the peak overshoot is greatly improved from Figure 30, the long-lasting high-frequency ringing indicates that the system is on the edge of instability. The cause of this ringing is the resonant peak in Figure 33 and the absence of gain margin in Figure 34. The frequency of the ringing and of the resonant peak indicate a minor-loop instability.

\section{B. Compensation with Minor-Loop Stability}

The problem with the compensation network in Figure 31 is that the minor loop is nearly unstable, which can be

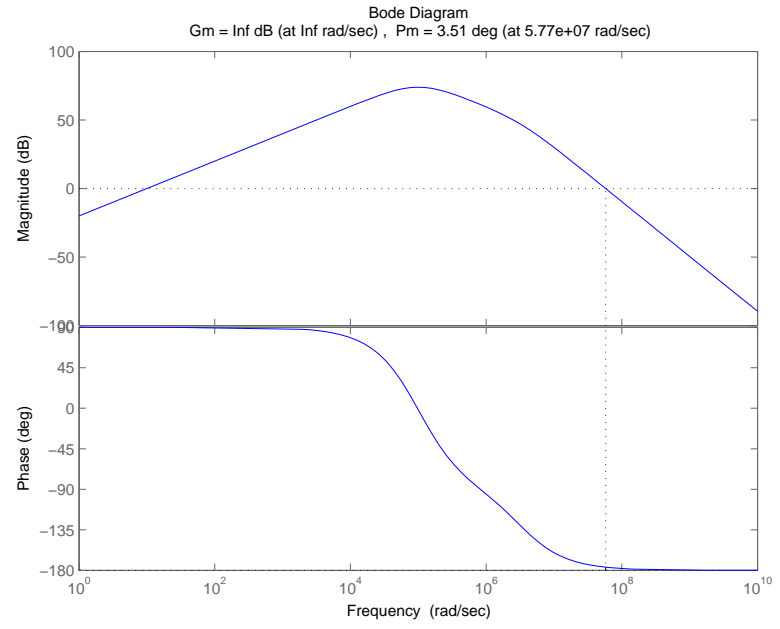

Fig. 36. Bode plot of the minor-loop transfer function $L_{m}(s)$ for the op amp with the compensation network from Figure 31. The pole in the admittance $Y_{c}(s)$ becomes a zero in $A(s)$, but the pole appears in the minor-loop transfer function and degrades the stability of the minor loop.

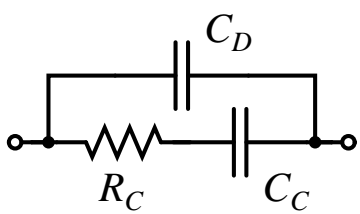

Fig. 37. Compensation network with a shunt capacitance. The capacitor $C_{D}$ introduces a zero into the minor-loop transfer function that can be used to increase the stability of the minor loop.

demonstrated by examining the minor-loop transfer function. As shown in the block diagram in Figure 25, the minor-loop transfer function is

$$
L_{m}(s)=G_{a}(s) Y_{c}(s) .
$$

The Bode plot of this minor-loop transfer function is shown in Figure 36. The admittance pole from the compensation network appears directly in the minor-loop transfer function and degrades the stability of the minor loop.

To improve the stability the minor loop, the compensation network is augmented with a shunt capacitance [12] as shown in Figure 37. The capacitor $C_{D}$ introduces a zero into the minor-loop transfer function and is used to improve the minorloop phase margin. The compensation network admittance is

$$
Y_{c}(s)=\frac{s\left(C_{C}+C_{D}\right)\left(R_{C} C_{S} s+1\right)}{R_{C} C_{C} s+1}
$$

where

$$
C_{S}=\frac{C_{C} C_{D}}{C_{C}+C_{D}} .
$$

Using $R_{C} C_{S}=10^{-8}$ second, the Bode plot of the minorloop transfer function for the op amp with this improved compensation is shown in Figure 38. The additional zero from the capacitor $C_{D}$ increases the minor-loop phase margin to thirty-five degrees. 


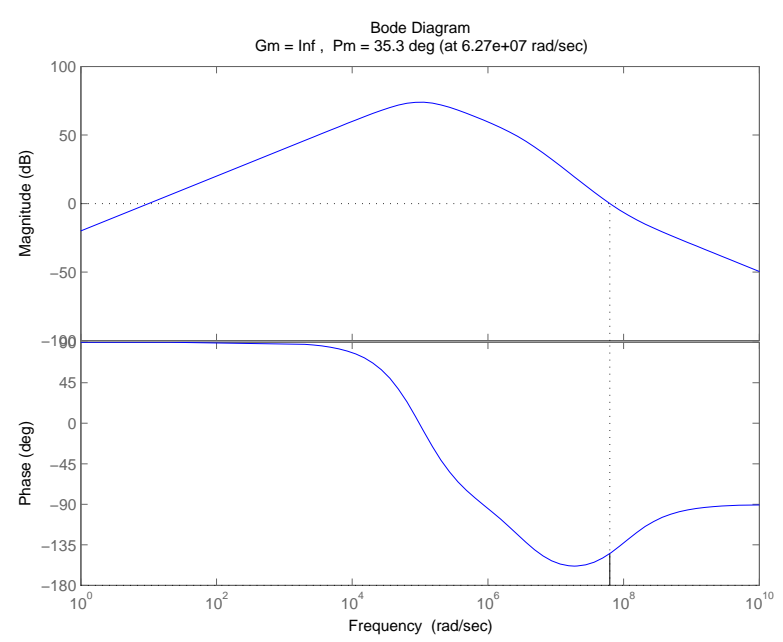

Fig. 38. Bode plot of the minor-loop transfer function $L_{m}(s)$ for the op amp with the compensation network from Figure 37. The additional zero from the capacitor $C_{D}$ increases the minor-loop phase margin to thirty-five degrees.

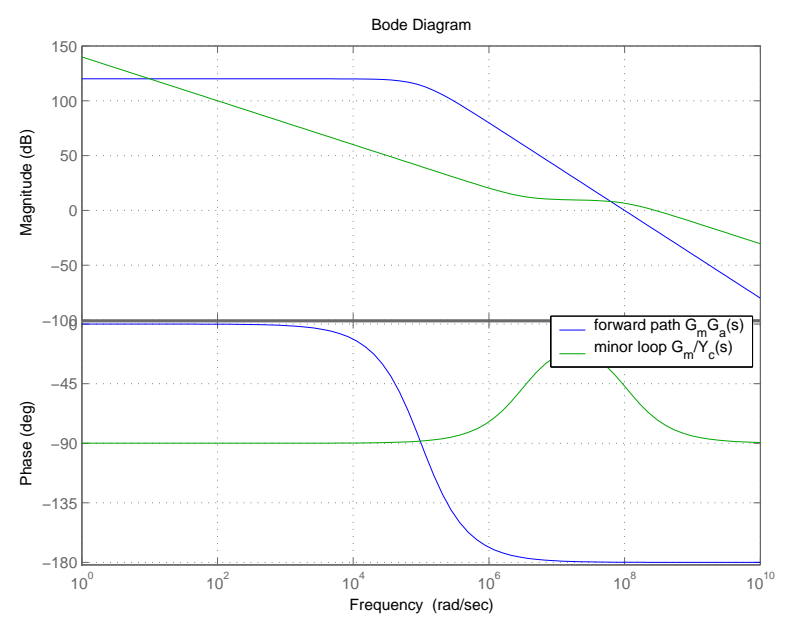

Fig. 39. Bode plot of the forward-path and inverse-feedback-path transfer functions for the op amp with the compensation network from Figure 37. The zero to cancel the capacitive-load pole is still visible near $3 \cdot 10^{6} \mathrm{rps}$. The pole from the capacitor $C_{D}$ appears beyond both minor-loop crossover and major-loop crossover at $10^{8} \mathrm{rps}$.

The Bode plot of the forward-path and inverse-feedbackpath transfer functions for the op amp are now shown in Figure 39. The zero to cancel the output capacitance pole is still visible at $3 \cdot 10^{6} \mathrm{rps}$. The pole from $C_{D}$ appears beyond both the major-loop crossover and minor-loop crossover frequencies.

The Bode plot of the op-amp transfer function is shown in Figure 40. The resonance from the minor loop has been greatly reduced compared to Figure 33 .

The Bode plot of loop transfer function for op-amp circuit with capacitive load is shown in Figure 41. In contrast to Figure 34, the loop transfer function now has adequate gain margin as well as sixty degrees of phase margin.

The step response of the compensated op-amp buffer with capacitive load is shown in Figure 42. The peak overshoot

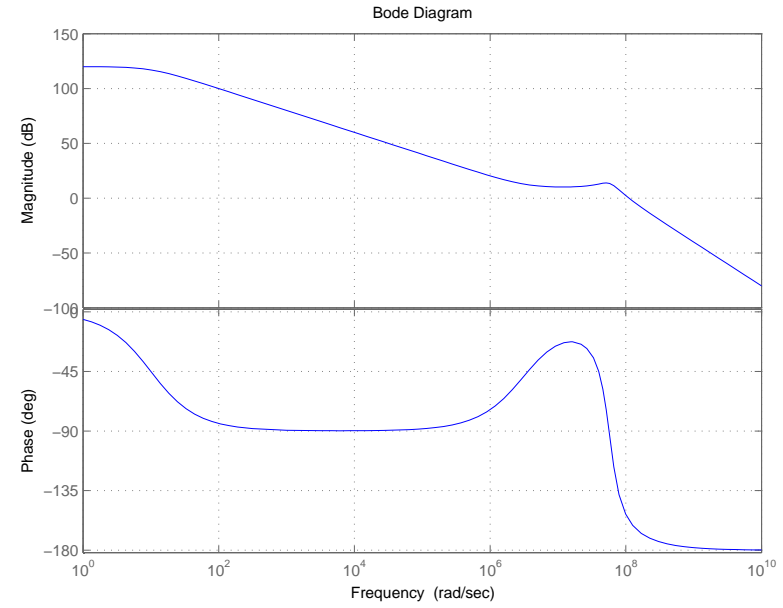

Fig. 40. Bode plot of the closed-minor-loop transfer function of the op amp $A(s)$. The resonance from the minor loop has been greatly reduced from Figure 33.

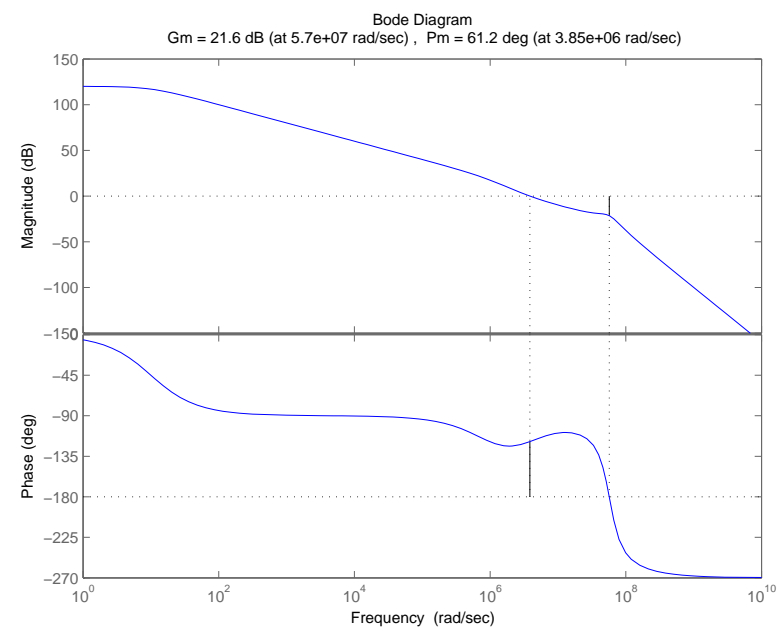

Fig. 41. Bode plot of the loop transfer function $L(s)$ for the op-amp buffer with capacitive load. In contrast to Figure 34, the loop transfer function now has adequate gain margin.

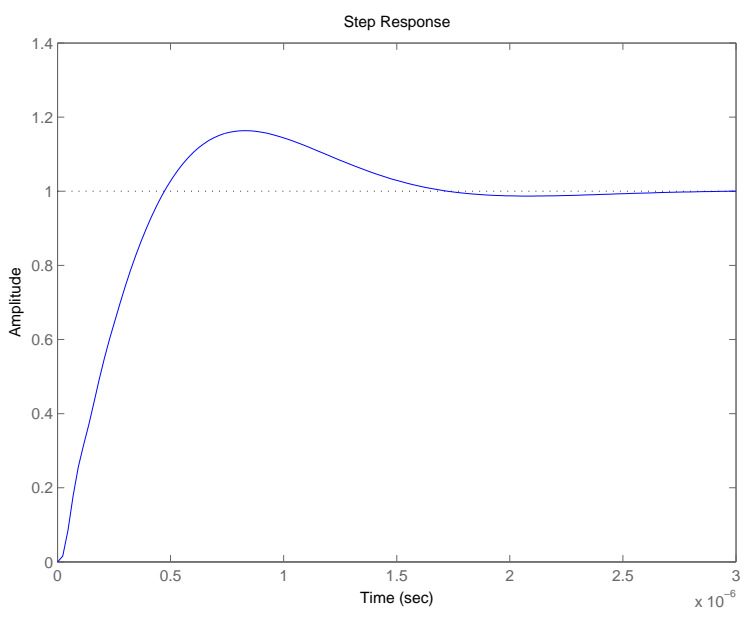

Fig. 42. Step response of the compensated op-amp buffer with capacitive load. The peak overshoot from Figure 30 is greatly reduced, and the highfrequency ringing from Figure 35 is gone. 


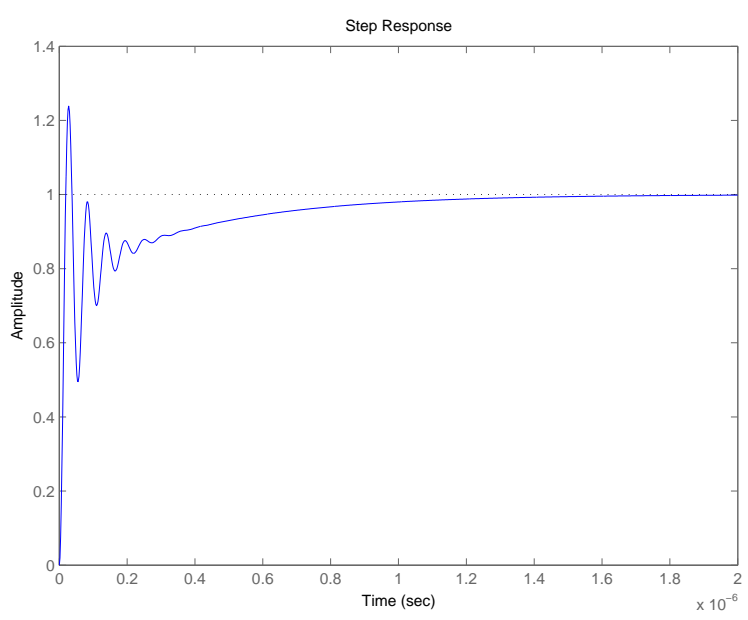

Fig. 43. Step response of the compensated op-amp buffer with the capacitive load removed. The special-purpose compensation developed here requires the capacitive load to be present for proper loop behavior. Without the capacitive load, the loop transfer function of the circuit is the transfer function of the op amp alone from Figure 40, which does not have adequate phase margin.

from Figure 30 is greatly reduced, and the high-frequency ringing from Figure 35 is gone.

As a final note, the step response of the compensated op-amp buffer with the capacitive load removed is shown in Figure 43. This special-purpose compensation developed here requires the capacitive load to be present for proper loop behavior. Without the capacitive load, the loop transfer function of the circuit is the transfer function of the op amp alone from Figure 40, which does not have adequate phase margin. However, with the capacitive load, the compensated op amp performs quite well.

Using the feedback approach to op-amp compensation design helped diagnose and solve the minor-loop stability problem. Without this approach, the solution to the highfrequency ringing in Figure 35 would have been extremely difficult to determine using direct circuit analysis techniques.

\section{CONCLUSIONS}

In all applications, op amps require a deliberately designed frequency response to ensure stability and satisfactory transient performance. Standard frequency compensation, using a capacitor around the high-gain stage, is designed for generalpurpose op-amp applications such as amplifiers, buffers, and integrators. Sophisticated compensation techniques can be employed in specific applications in which standard compensation methods perform poorly.

These compensation techniques are necessary to understand in the design of internally compensated op amps or in the use of externally compensated op amps. A pole-splitting approach to the compensation design is harmful to understanding. All of these techniques can be easily understood in a simple classical-control framework. Using a feedback approach to the compensation network design, insight and intuition into the behavior and flexibility of the system are gained.

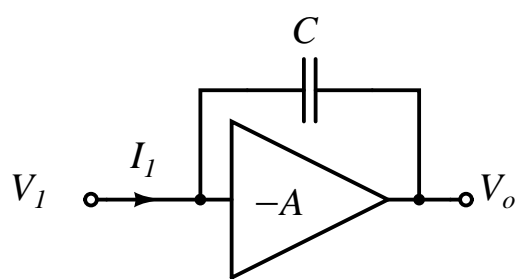

Fig. 44. Close-up of Figure 9 showing the compensation around the highgain second stage. The effective input impedance of this stage is modified by the Miller effect.

\section{APPENDIX I \\ MILLER COMPENSATION}

The Miller effect is the apparent scaling of an impedance connected from input to output of a gain stage, which was first noticed in vacuum tubes [14]. The input current into the second stage, as shown in Figure 44, depends on the total voltage across the capacitor

$$
I_{1}=\left(V_{1}-V_{o}\right) s C=V_{1}(1+A) s C .
$$

Thus for an amplifier with a large negative gain, the effective input capacitance appears $(1+A)$ times larger than the capacitor $C$.

From this effective capacitance, the low-frequency pole of the equivalent-circuit in Figure 10 can be estimated as

$$
\omega_{1}=\frac{1}{R C_{\text {eff }}}=\frac{1}{R_{1}\left(1+A_{2}\right) C} \approx \frac{1}{R_{1}\left(G_{M 2} R_{2}\right) C}
$$

which agrees with equation (4). For this reason, op-amp compensation with a capacitor around the second gain stage, as shown in Figure 8, is often called "Miller compensation."

\section{APPENDIX II \\ FAIRCHILD $\mu$ A741 COMPLETE SCHEMATIC}

The complete schematic for the Fairchild Semiconductor $\mu$ A741 operational amplifier is shown in Figure 45. This topology is classic and simple.

The primary signal path is comprised of three blocks. The first stage is the differential quad of transistors $Q_{1}-Q_{4}$ with active current-mirror load $Q_{5}-Q_{7}$. The second stage is the Darlington common-emitter amplifier $Q_{16}$ and $Q_{17}$ with current source load $Q_{13}$. A push-pull emitter-follower output buffer is implemented by transistors $Q_{14}, Q_{20}$, and $Q_{22}$.

The remaining transistors provide biasing and protection. The network of current mirrors $Q_{8}-Q_{13}$ produce bias currents for the transistors in the signal path. Compensation of the output-buffer dead-zone region is provided by $Q_{18}$ and $Q_{19}$. Output-current limiting and short-circuit protection is implemented by $Q_{15}$ and $Q_{21}-Q_{25}$.

And of course, the frequency compensation is accomplished by the $30 \mathrm{pF}$ capacitor around $Q_{16}$ and $Q_{17}$, as discussed in Section II. 


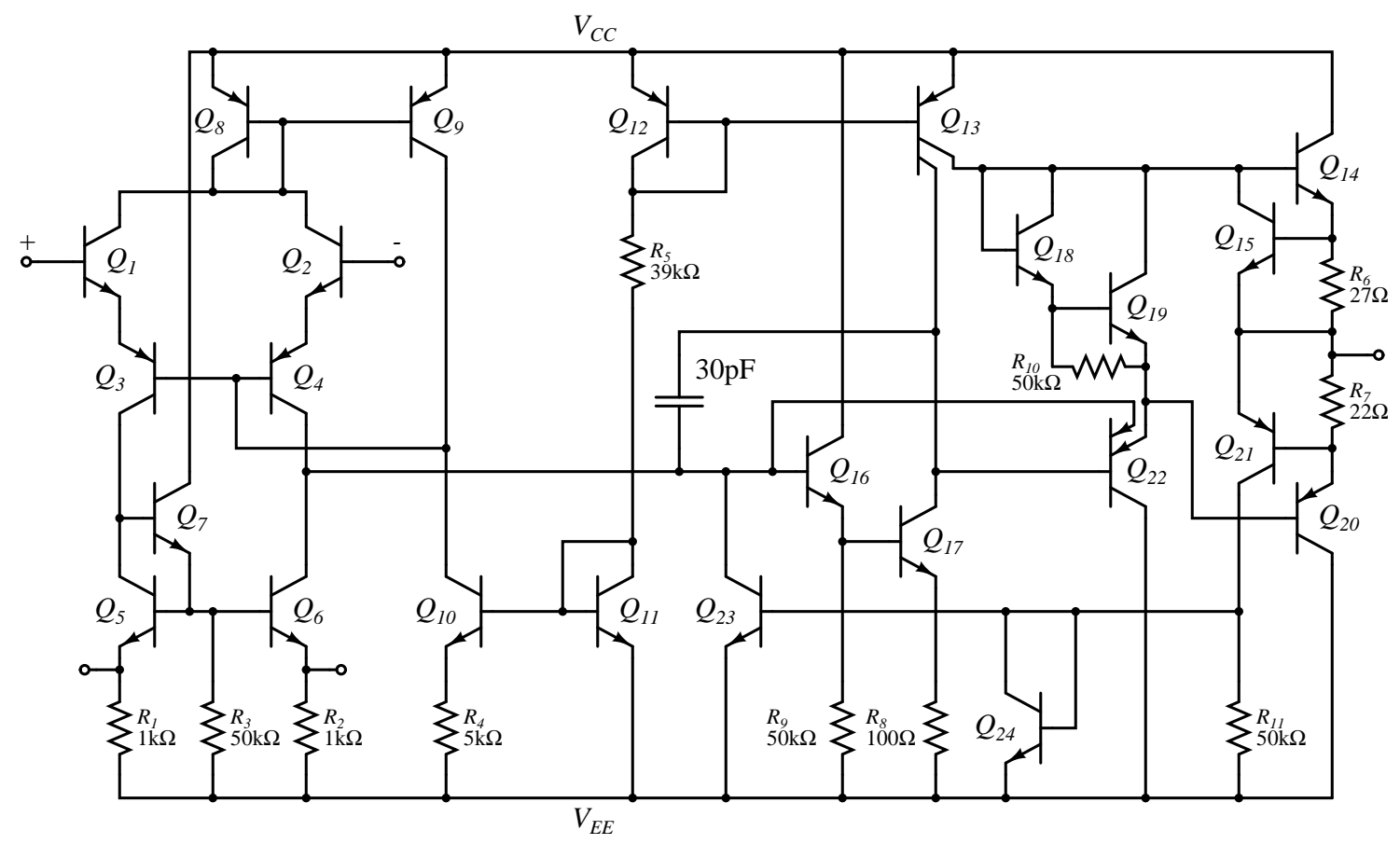

Fig. 45. Complete Fairchild $\mu \mathrm{A} 741$ schematic [10]. The primary signal path is comprised of two gain stages. The first gain stage is a differential pair with active load (transistors $Q_{1}-Q_{6}$ ). The second gain stage is a common-emitter amplifier (transistors $Q_{16}$ and $Q_{17}$ ). The output buffer is a push-pull emitter follower (transistors $Q_{14}, Q_{20}$, and $Q_{22}$ ).

\section{APPENDIX III \\ EXACT TRANSFER FUNCTION MATH}

The exact transfer function for the equivalent circuit in Figure 10 can be found directly from the node equations (2) and (3). Solving the output-node equation (3) for $V_{1}$

$$
\begin{gathered}
V_{o}\left(s C+\frac{1}{R_{2}}+s C_{2}\right)=V_{1}\left(s C-G_{M 2}\right) \\
V_{o}\left(R_{2}\left(C_{2}+C\right) s+1\right)=V_{1}\left(R_{2} C s-G_{M 2} R_{2}\right) \\
V_{1}=\left(\frac{R_{2}\left(C_{2}+C\right) s+1}{R_{2} C s-G_{M 2} R_{2}}\right) V_{o} .
\end{gathered}
$$

Massaging the input-node equation (2)

$$
\begin{gathered}
G_{M 1} V_{i n}+s C V_{o}=V_{1}\left(\frac{1}{R_{1}}+s C_{1}+s C\right) \\
G_{M 1} R_{1} V_{i n}+R_{1} C s V_{o}=\left(R_{1}\left(C_{1}+C\right) s+1\right)\left(\frac{R_{2}\left(C_{2}+C\right) s+1}{R_{2} C s-G_{M 2} R_{2}}\right) V_{o} \\
G_{M 1} R_{1} V_{i n}=V_{o}\left(\frac{\left(R_{1}\left(C_{1}+C\right) s+1\right)\left(R_{2}\left(C_{2}+C\right) s+1\right)}{R_{2} C s-G_{M 2} R_{2}}-R_{1} C s\right) .
\end{gathered}
$$

Solving for the transfer function

$$
\begin{gathered}
\frac{V_{o}}{V_{i n}}(s)=\frac{G_{M 1} R_{1}\left(R_{2} C s-G_{M 2} R_{2}\right)}{\left(R_{1}\left(C_{1}+C\right) s+1\right)\left(R_{2}\left(C_{2}+C\right) s+1\right)-\left(R_{2} C s-G_{M 2} R_{2}\right) R_{1} C s} \\
\frac{V_{o}}{V_{i n}}(s)=\frac{G_{M 1} R_{1} G_{M 2} R_{2}\left(C s / G_{M 2}-1\right)}{R_{1} R_{2}\left(C_{1} C_{2}+C_{1} C+C_{2} C\right) s^{2}+\left(R_{1} C_{1}+R_{1} C+R_{2} C_{2}+R_{2} C+G_{M 2} R_{2} R_{1} C\right) s+1} .
\end{gathered}
$$

This second-order transfer function is the expected result from the topology in Figure 10. All of this math can be avoided using the feedback techniques in Section IV and Appendix V. 


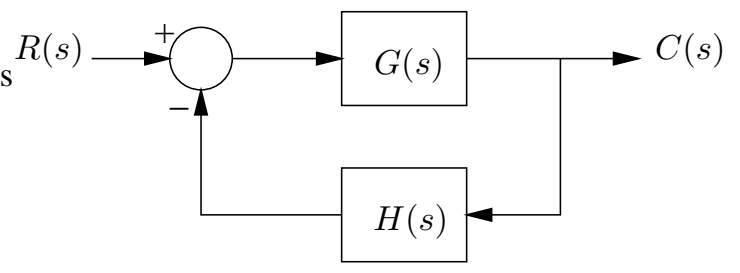

Fig. 46. General block diagram for a feedback system. Standard notation uses $R(s)$ for the reference input, $C(s)$ for the controlled output variable, $G(s)$ for the forward-path transfer function, and $H(s)$ for the feedback-path transfer function.

\section{APPENDIX IV \\ Closed-Loop Transfer FunCTION TRICK}

The work for finding an approximate transfer function of a feedback system, such as shown in Figure 46, can be simplified by taking advantage of a useful trick involving Black's formula

$$
\frac{C}{R}(s)=\frac{G(s)}{1+G(s) H(s)} .
$$

Black's formula can be simplified when

$$
|G(s) H(s)| \gg 1
$$

or equivalently, when

$$
|G(s)| \gg \frac{1}{|H(s)|} .
$$

When this inequality is true, the $G H$ term dominates in the denominator, and the magnitude of the closed-loop transfer function can be rewritten as

$$
\left|\frac{C}{R}(s)\right|=\left|\frac{G(s)}{1+G(s) H(s)}\right| \approx \frac{1}{|H(s)|} .
$$

Similarly, when

$$
|G(s) H(s)| \ll 1
$$

or equivalently, when

$$
|G(s)| \ll \frac{1}{|H(s)|}
$$

the unity term dominates in the denominator. The magnitude of the closed-loop transfer function is then approximately

$$
\left|\frac{C}{R}(s)\right|=\left|\frac{G(s)}{1+G(s) H(s)}\right| \approx|G(s)| .
$$

Therefore, the asymptotic frequency response of a closedloop feedback system, can be plotted by graphing $|G(s)|$ and $|H(s)|^{-1}$ and then tracing the lower curve for all frequencies.

\section{APPENDIX V \\ Right Half-Plane Zero}

In the analysis in Section IV, the effect of the voltage $V_{1}$ on the current through the compensation capacitor was ignored. Unfortunately, the output voltage of the first stage, while indeed small, is not zero. Both currents that flow through the compensation capacitor are shown in Figure 47, where

$$
I_{c}=s C V_{o}
$$

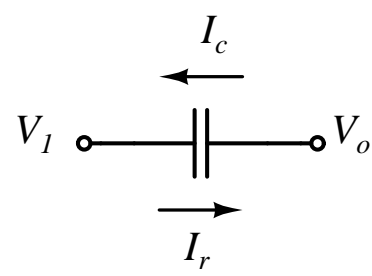

Fig. 47. The two currents that flow through the compensation capacitor. The feedback current $I_{c}$ is the compensation current discussed in Section IV. The feedforward current $I_{r}$ causes a right half-plane zero in the op-amp transfer function.

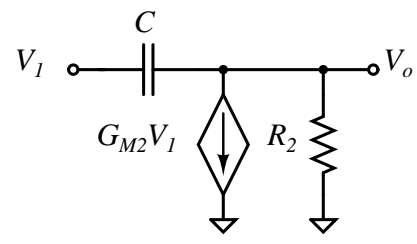

Fig. 48. Close-up of Figure 10 showing the effect of the feedforward current $I_{r}$ on the second stage. The output voltage is zero when the total current flowing into the output stage is zero.

and

$$
I_{r}=s C V_{1} .
$$

The feedback current $I_{c}$ is the compensation current discussed in Section IV. The feedforward current $I_{r}$ causes a right halfplane zero in the op-amp transfer function. By superposition, the sum of these two currents is the total current flowing in the compensation capacitor.

A close-up of the effect of the feedforward current $I_{r}$ on the second stage is shown in Figure 48. The output voltage is zero when the total current flowing into the output stage is zero, that is

$$
s C V_{1}-G_{M 2} V_{1}=0 .
$$

This zero occurs at a frequency

$$
\omega_{z}=+\frac{G_{M 2}}{C} .
$$

Therefore equation (7) must now be written as

$$
V_{o}=\left(-G_{M 2} V_{1}+s C V_{1}\right) \frac{R_{2}}{R_{2} C_{4} s+1} .
$$

The complete block diagram of the op-amp equivalent circuit, including the feedforward current through the compensation capacitor, is shown in Figure 49. The parallel blocks in the forward path can be collapsed into a single block

$$
-G_{M 2}+s C=-G_{M 2}\left(1-\frac{s C}{G_{M 2}}\right) .
$$

Thus the feedforward current causes a right half-plane zero. The negative phase shift from this right half-plane zero can place considerable limits on op-amp performance.

This problem can be fixed. A compensation network to cancel the right half-plane zero is shown in Figure 50 with admittance

$$
Y_{c}(s)=\frac{I_{c}}{V_{o}}=\frac{C s}{R C s+1} .
$$




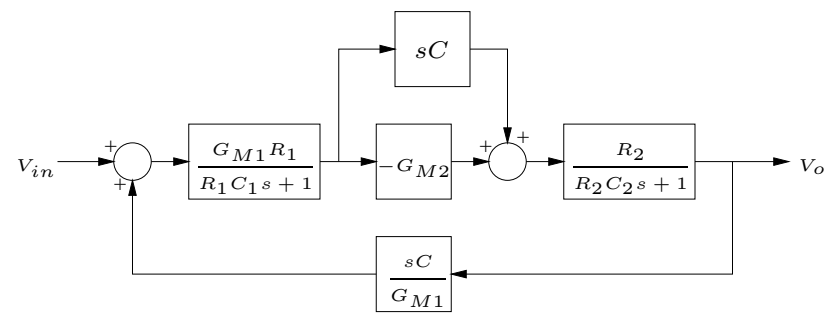

Fig. 49. Block diagram of the op-amp equivalent circuit, including the feedforward current through the compensation capacitor. The feedforward term causes a right half-plane zero in the op-amp transfer function.

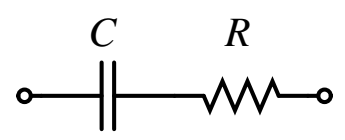

Fig. 50. Compensation network to cancel the right half-plane zero due to the feedforward current through the compensation capacitor.

The compensating admittance $Y_{c}(s)$ shows up in both the feedback path and the feedforward path. The block in the forward path becomes

$$
\begin{aligned}
-G_{M 2} & +\frac{C s}{R C s+1}=\frac{-G_{M 2} R C s-G_{M 2}+C s}{R C s+1} \\
& =-G_{M 2} \frac{\left(R-1 / G_{M 2}\right) C s+1}{R C s+1} .
\end{aligned}
$$

Thus, for a choice of $R=1 / G_{M 2}$, the zero moves out of the right half-plane to infinity.

The right half-plane zero is usually not a problem in bipolar op amps. For example, in the $\mu \mathrm{A} 741$

$$
f_{z}=\frac{G_{M 2}}{2 \pi C}=\frac{6.8 \mathrm{~m} \mho}{2 \pi 30 \mathrm{pF}}=36 \mathrm{MHz} .
$$

In a CMOS op amp, where transistor transconductances can be much lower, the right-half plane zero frequency can be quite close to the unity-gain frequency of the op amp.

\section{ACKNOWLEDGEMENTS}

Special thanks to James Roberge for many helpful discussions and for comments on this paper. Thanks to Daniel Abramovitch, Jesse Bankman, Stefano D'Aquino, Joel Dawson, and Rahul Sarpeshkar for helpful comments. Double thanks to Daniel Abramovitch for inviting this tutorial and for his inspirational paper title [15].

\section{REFERENCES}

[1] J. R. Ragazzini, R. H. Randall, and F. A. Russell, "Analysis of problems in dynamics by electronic circuits," Proceedings of the IRE, vol. 35, pp. 444-452, May 1947.

[2] D. H. Sheingold, Ed., Applications Manual for Operational Amplifiers for Modeling, Measuring, Manipulating, and Much Else. Boston: George A. Philbrick Researches, 1965.

[3] G. A. Korn and T. M. Korn, Electronic Analog Computers. New York: McGraw-Hill, 1952.

[4] J. S. Small, The Analogue Alternative: The Electronic Analogue Computer in Britain and the USA, 1930-1975. London: Routledge, 2001.

[5] R. J. Widlar, "Monolithic op amp with simplified frequency compensation," EEE, vol. 15, pp. 58-63, July 1967.

[6] P. R. Gray and R. G. Meyer, Analysis and Design of Analog Integrated Circuits. New York: Wiley, 1993.

[7] D. A. Johns and K. W. Martin, Analog Integrated Circuit Design. New York: Wiley, 1996.

[8] R. Gregorian, Introduction to CMOS Op-Amps and Comparators. New York: Wiley, 1999.

[9] J. E. Solomon, "The monolithic op amp: a tutorial study," IEEE Journal of Solid-State Circuits, vol. 9, pp. 314-332, Dec. 1974.

[10] D. Fullagar, "A new high performance monolithic operational amplifier," Fairchild Semiconductor, Application Brief, May 1968.

[11] K. H. Lundberg, "Pole splitting considered harmful," April 23, 2003, ECE Colloquium, Department of Electrical and Computer Engineering, University of Massachusetts Dartmouth.

[12] J. K. Roberge, Operational Amplifiers: Theory and Practice. New York: Wiley, 1975.

[13] R. J. Widlar, "Monolithic operational amplifiers the universal linear component," National Semiconductor Corporation, Application Note AN-4, Apr. 1968.

[14] J. M. Miller, "Dependence of the input impedance of a three-electrode vacuum tube upon the load in the plate circuit," Scientific Papers of the Bureau of Standards, vol. 15, no. 351, pp. 367-385, 1920.

[15] D. Y. Abramovitch, "Phase-locked loops: A control centric tutorial," in Proceedings of the 2002 American Control Conference, Anchorage, Alaska, May 2002. 